\title{
Heat based Odors Delivery Apparatus for Interactive Game Playing
}

\author{
Saad Hameed Abid ${ }^{1,2}$, Zhiyong $\mathrm{Li}^{1}$, Renfa $\mathrm{Li}^{1}$ and Jumana Waleed ${ }^{3}$ \\ ${ }^{1}$ College of Computer Sciences and Electronic Engineering, \\ Hunan University, Changsha, Hunan 410082, China \\ ${ }^{2}$ AL-Mansour University College, Al-Andalus square, Baghdad, Iraq \\ ${ }^{3}$ School of information science and engineering, Central South University, \\ Changsha, 410083, China \\ saad@hnu.edu.cn ${ }^{1}$,zhiyong.li@hnu.edu.cn ${ }^{1}$, \\ lirenfa@hnu.edu.cn jumana@csu.edu.cn ${ }^{3}$
}

\begin{abstract}
Scent delivery systems or what are known as olfactory displays has been the most recent trend in human computer interaction, control and automation, virtual reality, augmented reality, e-commerce and many other fields in the computer world. These devices have the ability to generate smell combinations for some digital items in the computer virtual world. The idea is to deliver additional perception (olfactory perception) to the user to enrich his/her indulgence while interacting with the computer. The proposed olfactory display is similar to this concept but greatly differ in many aspects, using laser based atomization method to produce enough sudden heat to generate odors that cannot be generated using chemical materials these odors are smoke, gun powder, wood milling, cigarettes smoking, plastic melting and Incense. All olfactory displays researchers avoid involving these types of odors in their studies due to the difficulty of the generation and control of such odors and the absence of their correct and precise generation using chemical materials. The proposed system will provide a way of generating such odors while interactive game is being played by a user with minimum impact on the environment to avoid adaption to the user's olfactory system and polluting the environment that the user occupies.
\end{abstract}

Keywords: Olfactory displays, scent, odor, smell, games

\section{Introduction}

The trend towards better human-computer interaction has grown in the past few years. Yet the researches main focuses are on human visual and auditory systems and some haptic manipulations, human olfactory system has some researches also, but compared to other human-computer interaction researches is considered very low and did not draw its rightful attention by researchers. On the other hand, interactive game industry has a huge attention due to its high revenue in the world market drawing many companies to invest significant amount of resources which welcomes new age technologies to be involved in the process to target wider markets. Regarding the proposed system to the attempt develop a complete solution, concerning all aspect in olfactory displays field for the purpose of satisfying most constraints resulting best outcome. This paper will discuss the existing scent delivery techniques and improvements in the related works section, followed by the design concept section which shows the implications and details of the proposed scent delivery device focusing on practicality and simplicity, experimental results are shown afterwards the experimental results are presented in the experimental 
results section, and finally the conclusion drawn from the work is presented in the conclusions section.

\section{Related Works}

Regarding the scent delivery device, there are many researches that focus on the scent delivery mechanisms itself using existing air pumps to disperse the scent into air and solenoid valves to control its amount as seen in [1], improving this research to use a rapidly switching valves to cope with required speed and improve the scent delivery is proposed in [2] and the same concept is used in a video has been proposed in [3] as an application for the proposed technique in olfactory displays. Other improvements on such concept of scent delivery is presented in [4] using computational fluid dynamics (CFD) for vivid scent delivery. Hirose et al. introduced number head-mounted olfactory displays, including a scent generation and blending mechanism controlled by computer [5,6]. They recently developed a wearable olfactory display system [7] that allows users to move freely without being tied to the system by tubes and wires. In these display systems, scented air was introduced to the nose through a tube. The visual display counterpart to such olfactory interfaces is, of course, (human mounted displays) HMD. Mochizuki et al. developed an arm-worn olfactory display that focuses on the human habit of grasping a target object with a visual tag, bringing it up to the nose, and sniffing it $[8,9]$. also in [10] it uses a micro pump to deliver the scented material onto a SAW (surface acoustic wave) device it has promising results but the hardware setup is not practical and requires voltage levels as high as 100 volts for the driving circuit not to mention a special laboratory environment. Other olfactory displays were presented using inkjet technology using a modified inkjet cartridge to hold the scent data and produce it using different level of intensity achieving high control over the amount and speed but the chemical properties of the scented materials are altered due to heat used to eject the scent as in [11]. Game playing improvements are not limited to olfactory displays only, other improvements such as [12] and [13] tackled users' emotions and haptic sensory. Regardless of the varying techniques and technologies to deliver the scent to the user it always consists of a liquid scented material (oil, water or alcohol based) diluted in another dispersing material such as water or alcohol. This will put a limitation of representing some scents/odors that cannot be simulated using liquid based materials especially if the scent/odor is heat related and mentally represented in humans' mind as so.

\section{Design oncept}

The design of the proposed olfactory display depends on some constraints; this is because of the challenges of providing a heat source (laser diode) and an exchangeable target for that heat source in addition to a controlled air flow to achieve delivery speed and prevents discomfort of continuous air to the users face. The following is the explanation of each part design and behavior in the olfactory display.

\subsection{Rotatable Scent Cartridge}

The rotatable scent provision mechanism is a hexagon shaped cartridge with each face sized $34 \times 20 \mathrm{~mm}$ having square cavities to hold the solid scent materials each cavity is sized $31.5 \times 10 \times 4 \mathrm{~mm}$. This hexagon rotates in both directions to provide the desired solid scent material to be placed in front of the laser beam with speed as high as $60 \mathrm{rpm}$. Each time a point at the scented material is targeted the hexagon rotates this point away to allow a fresh point to be targeted by the laser this is to provide highest scent release to take place. The reason is due to the lased has a fixed focal point and the hexagon shape 
while rotation enables this focal point to intersect with the scent materials in several places and depths ensuring that the scent materials to be burned by the laser in various focal points and locations. If there are no scents to deliver the hexagon rotates 5 times at highest speed to expel all the residual material left behind (manly carbon or soot) by a previous scent ejection, see Figure 1.

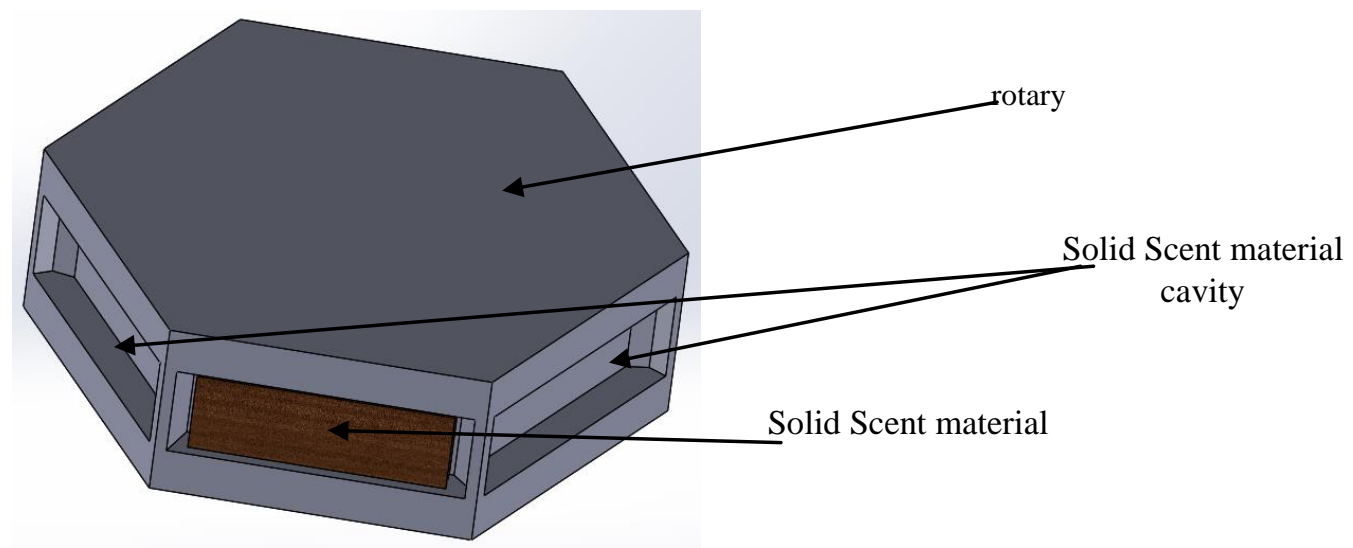

Figure 1. Hexagon Rotary with Six Solid Scent Materials Mounted to its Faces

\subsection{Laser Diode}

Using a $1000 \mathrm{mw} 660 \mathrm{~nm}$ laser is suitable for the proposed application, it has a stable beam that can produce enough heat to produce the desired smell, and the laser is placed with distance enough to get the best focal point of its lens, see Figure 2. The laser pulses supported by the laser driver ( $2.3 \mathrm{v}, 2 \mathrm{amps})$ and controlled by the microcontroller (both mounted underneath) are produced according to the desired position on the solid scent materials and based on a positioning algorithm stored in the microcontroller which will be explained later in this paper.

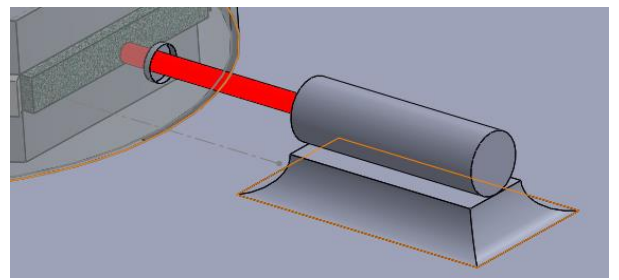

Figure 2. Laser Diode Hitting the Solid Scent Material

\subsection{Air Flow Fan}

The fan is controlled via pulse width modulation signal PWM produces by the microcontroller and amplified using a PNP transistor. The fan rotates at full speed of 2500 rpm and produces enough air flow that can reach the user's face sufficiently. The rotation of the fan forces the air to exit from the upper shaft creating a low pressure inside the housing which forces the air to flow from the laser hole carrying the smoke particles and cooling the burned area instantaneously, it is mounted above the hexagon rotary and rotates independently from it, the rotation speed of the fan varies according to the desired amount of the produced smell/odor and operation, see Figure 3. 


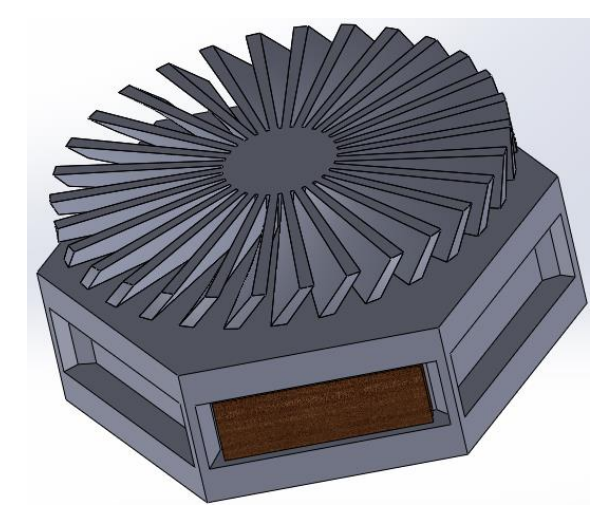

Figure 3. Air Flow Fan position

\subsection{Housing}

The housing is a transparent plastic containing two holes the upper hole is the smell/odor ejection point and the lower hole is the air entry hole and laser projection opening. It maintains the air flow inside the device, see Figure 4.

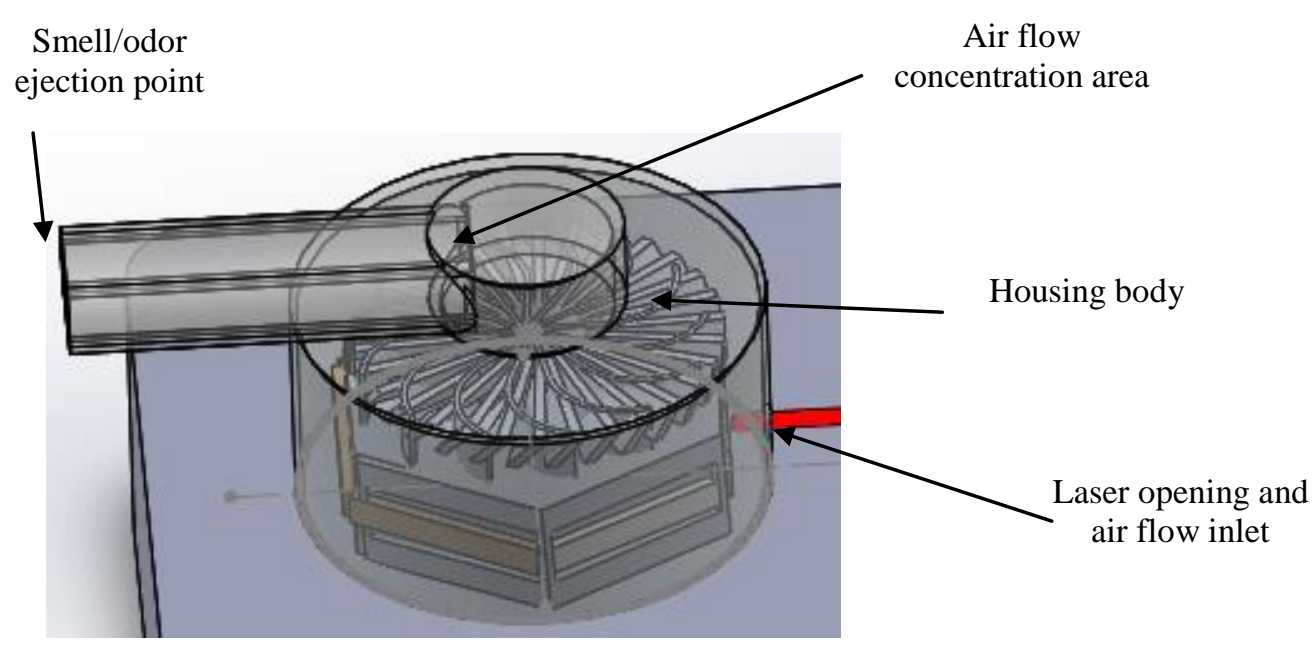

Figure 4. Housing of Scent Delivery Assembly

\subsection{Electronics and Motors}

Underneath the system the electronic boards are mounted, they consists of a microcontroller, driver circuits for the laser, the fan, and the stepper motor, and a stepper motor for the hexagon rotary for precise movement.

\section{Solid Scent Material Preparation}

The selected smells/odors are: wood milling, smoke, cigarettes, gun powder, incense and melting plastic. The actual materials that correspond to each smell/odor (except gun powder) is grinded to a coarse sized particles using a household coffee grinder and a sifter. An odorless binding material is used to bind the grinded materials, using polyvinyl acetate (PVA), also known as "white glue" to bind the materials with a 1:100 wood glue to water mix ratio is used and the final mixture is compressed using a custom compactor see Figure 5. Mild pressure is required to produce a solid block and dried for 24 hours to make sure that the block can withstand rotation speed and will not shatter accordingly. As for the plastic scent material, a rectangular piece of black colored polyurethane is used; it 
has some air bubbles which makes it suitable for high odor generation. Also for the gun powder odor a set of chemical materials were used to form a compound that produces a gunpowder like odor without combusting like a regular gunpowder which exhausts the whole cartridge in one use not to mention it is hazardous to use such materials indoors. Table 1 shows the proper mixture ratios and materials to produce a putty like compound that when heated by the laser produces the same odor or the burnt gun powder. These approaches provide an easy way to refill the scent/odor cartridge with minimum cost and effort in addition to lower impurities or contaminants in the produced scent/odor.

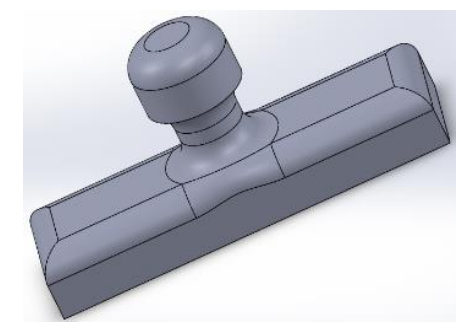

Figure 5. Compactor Used to Compact the Sent Material into the Cavity of the Hexagon

Table 1. Raw Materials Name and Weights in Grams to Generated Burnt Gun Powder Putty like Scent/odor Material

\begin{tabular}{|c|c|}
\hline Material name & Weight in grams \\
\hline Potassium permanganate & 5 \\
\hline Iron oxide & 0.2 \\
\hline Calcium carbonate & 53 \\
\hline Fumed silica & 1.8 \\
\hline Epoxy resin & 45 \\
\hline
\end{tabular}

\section{Software}

The game used is based on an implementation on Matlab that provides a 3D environment and the player can move freely in the designed maze, the ability to fire a gun, a machine gun was added to the program with sound and visual effects. Furthermore several scenes were added in several rooms creating the desired effect for our system. Several rooms contained a fire, a shrine, a saw mill with some wood stored in it and a non-hostile character smoking a cigarette that the user can come close to.

Each time the user shoots a round from the gun the signal is also sent to the proposed olfactory display, the signal contains a frame of data describing the event and the duration of time that this event should last along with the intensity of that event. The microcontroller rotates the hexagon with the desired scent/odor in front of the laser then start activating the laser with time duration delivered by the data frame arrived from the computer correspond to the event. Algorithm 1 shows the technique for scent/odor generation.

Algorithm 1: data frame handling

When data frame is received the following steps are executed to deliver the scent/odor to the user's olfactory system.

Step 1: parse the data frame and identify the desired scent and duration of delivery along with its intensity. 
Step 2: calculate the number of steps sent to the stepper motor from the current position to the desired position by both clockwise and counterclockwise, compare and use the least to move the stepper motor.

Step 3: activate the laser diode.

Step 4: rotate hexagon clockwise and counterclockwise with speed=duration/number of steps per face*5.

Step 5: increase fan speed to speed=intensity.

Step 6: disable the laser

Step 7: rotate the hexagon five full revolutions at highest speed.

Step 8: deactivate fan.

The algorithm uses a rocking effect to pass the solid scent material in front of the laser beam several times with different angles intersecting different focal points while letting the fumes and smokes to accumulate in the housing then activating the fan at full intensity speed to deliver the scent to the user. The back and forth rocking takes five times for each scent face. Finally, rotating the hexagon to expel all the residual remains from the burning process by centrifuge force.

When the user walks into a room with a certain scene if the scene carries some smell properties then the process of scent generation starts all over again depending on the distance of the user from the object inside the scene with the smell properties, Figure 6 show the distance correspondence to intensity amount sent to the display. Figure 7 show game layout with some scent/odor related events (top view) and Figure 8 shows scent/odor events in user prospective.

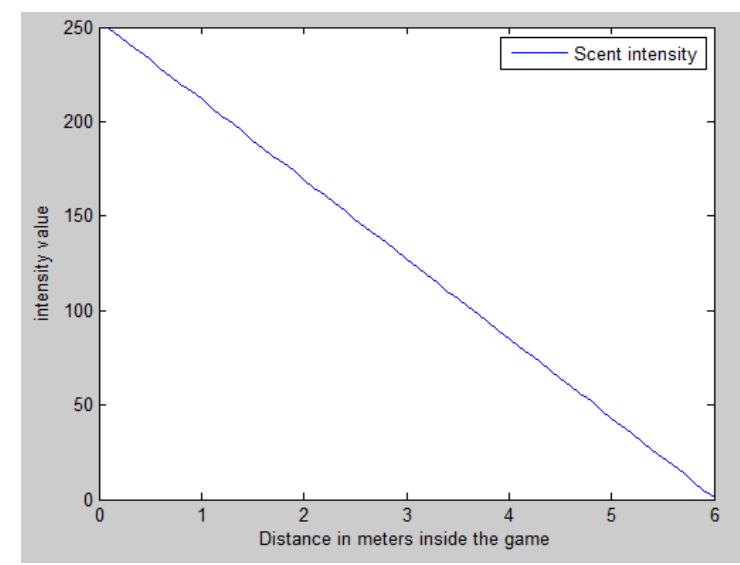

Figure 6. Intensity Values with Respect to Distance Inside the Game

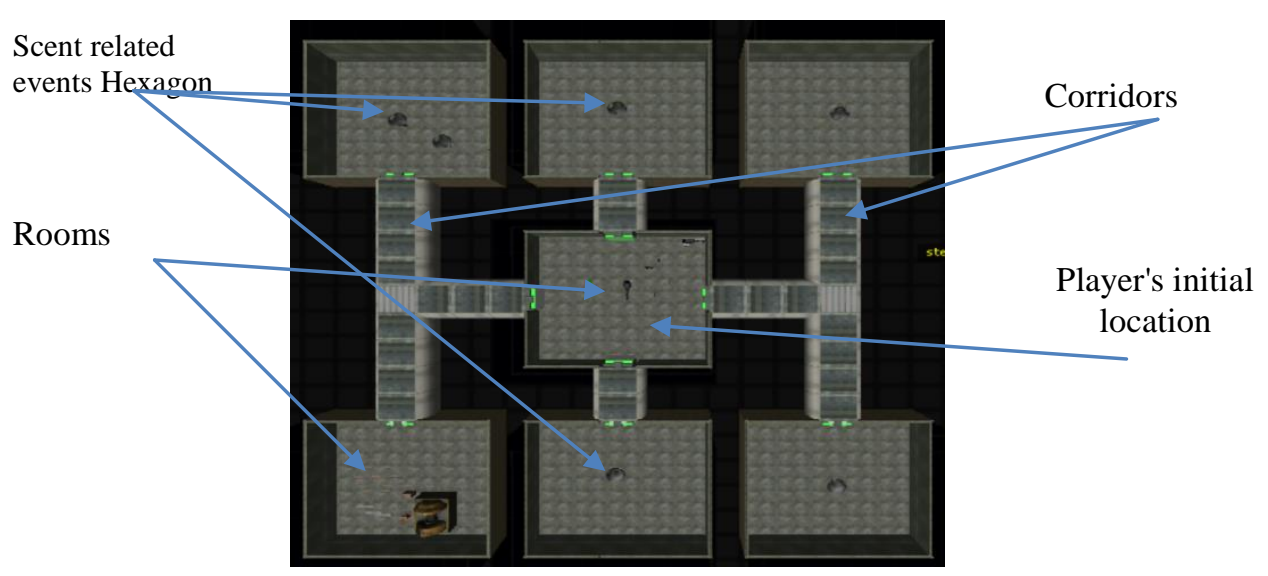

Figure 7. Game Layout with Some Smell/odor Events 


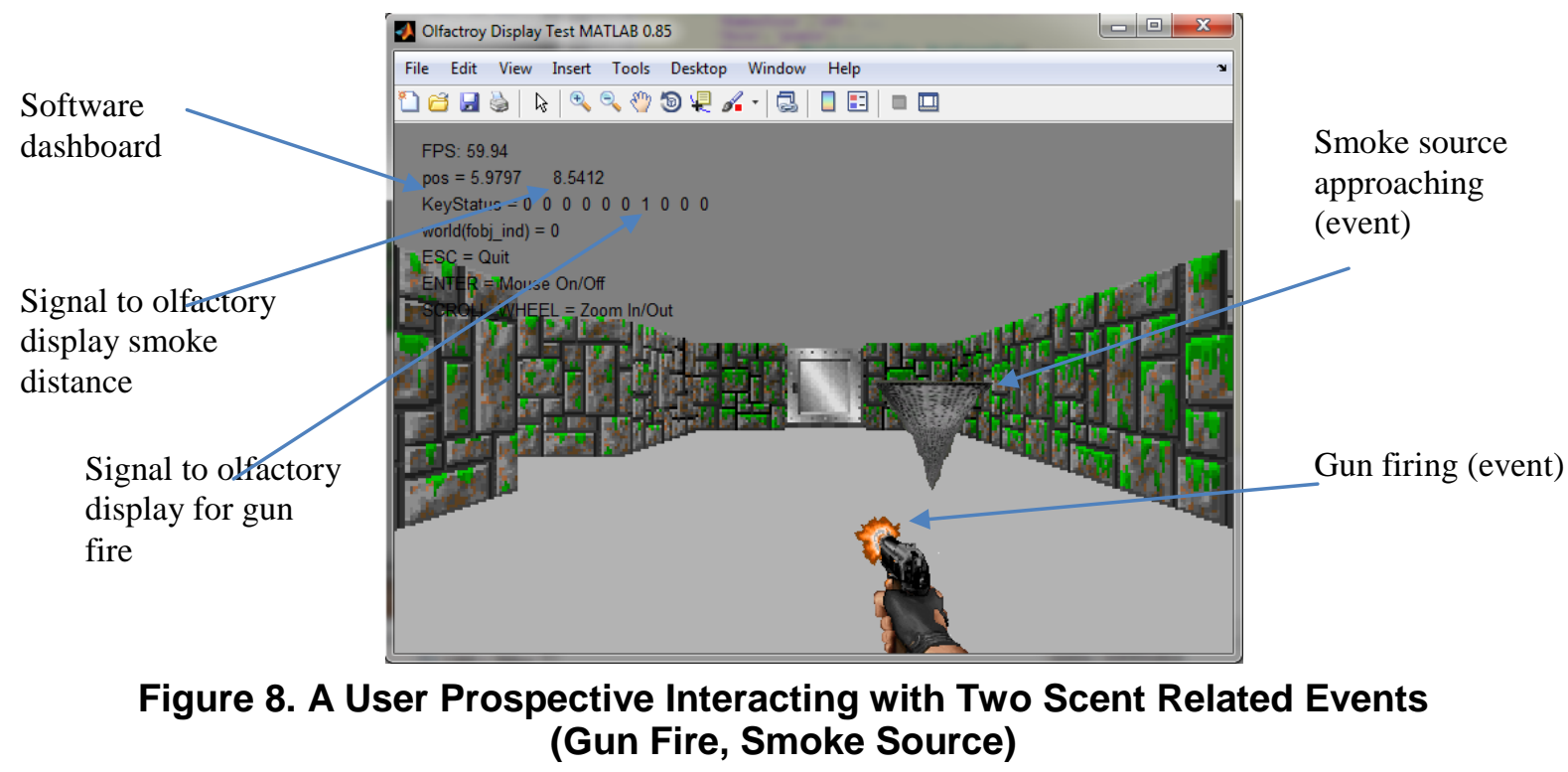

Each time and event persists the amount of intensity is determined by the system and sent to the olfactory display. Some cases the player inside the first person shooters game start walking towards an object with scent properties causing the software to successively send data fames with increasing intensity values to the olfactory display swamping the microcontroller with instructions and causing it to lag rendering the responsiveness of the display to be inacceptable. In addition a repeated shooting event or usage of rapid fire weaponry such as a machine gun from the player causes the olfactory display to receive also a large number of data frames causing it to swamp the microcontroller and substantially degrading its responsiveness. For such reasons the software uses algorithm 2 to prioritize the events and eliminates recurring data and optimizes utilization and inhibiting hexagon rotary five revolution expelling procedure.

Algorithm 2: pseudo code for optimizing events

For each 1 second time frame do

Get all scent related events

Prioritize scents according to following:

Gun powder (lowest)

(Smoke, cigarettes, incense) middle

(Melting plastic, Wood milling) high

End for

For each matching scent events combine events into one event adding both intensities and times

If result $>$ max intensity generate a new frame placing result modulo max intensity

End for

Re order frames

Send to olfactory display

The above algorithm optimizes frames for $1 \mathrm{sec}$ events to send to the olfactory display the number of frame is reduced by merging similar frames and prioritizing different frames in the mentioned order. 


\section{Experimental Results}

In a controlled environment some experimental results were obtained regarding the response time and delivery, a MQ-2 sensor were used and the ambient temperature was 23-25 degrees Celsius and humidity of $50-55 \mathrm{~g} / \mathrm{m} 3$ the sensor is 60 $\mathrm{cm}$ away from the olfactory display's ejection point, in initial tests a range of intensity values were sent consecutively to the olfactory display and the response values of ppm (particles per million) were registered, Figure 9 shows the relation between the ejected scent and received smell by the sensor.

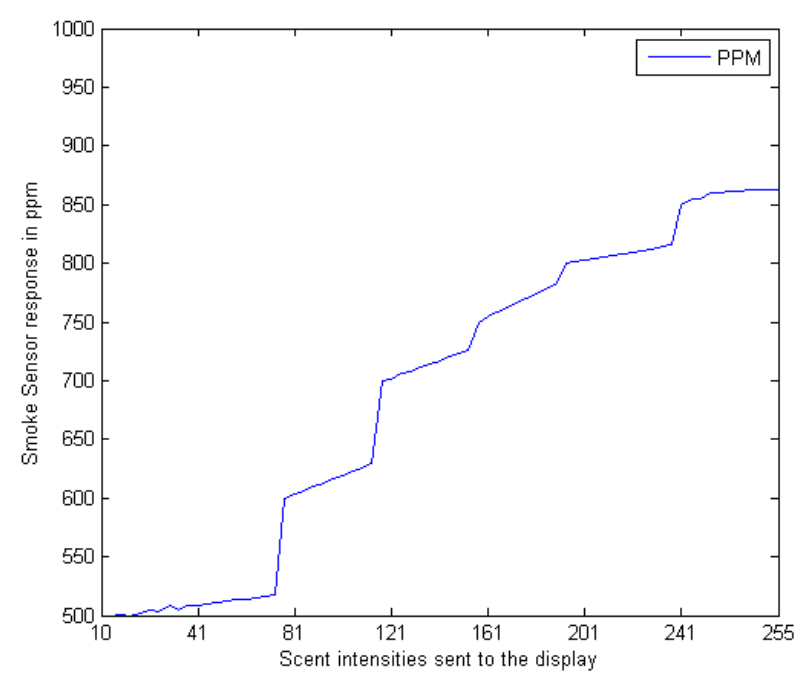

Figure 9. Measured ppm Values for Different Intensities

Regarding response time for the system, the same sensor was used to detect the first occurrence of the scent particles with a threshold value of $550 \mathrm{ppm}$ (around minimum), when the display receive a data fame it starts a timer and the response of the sensor stops that timer indicating a detection has occurred. This process is done for the six types of scents/odors. Since all odors are generated by a laser burning a certain material, there is always a smoke generated in the process, the smoke is dispersed in the air post ejection point and hardly visible by naked eyes but still detected by the sensor with different detection response values. These values are shown on Figure 10 for all scent materials in the system.

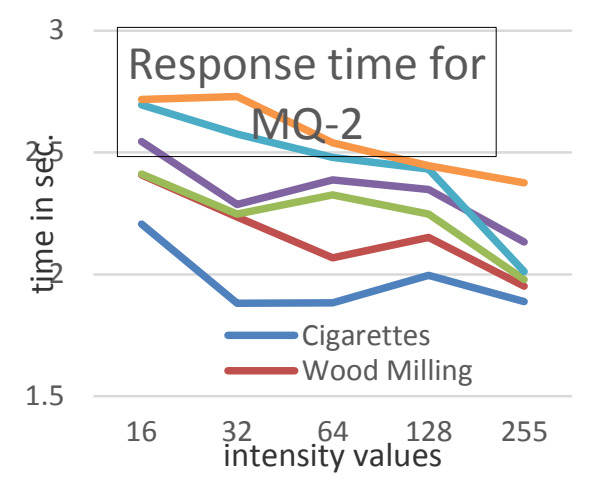

Figure 10. Response Time of MQ-2 Smoke Sensor for Six Different Scent/odors Materials 
The hexagon holds the materials in certain order; this order is dictated by Figure 10 . Placing each scent/odor material in a relation close to their detection times so the switching operation takes place fast. The order of the scent materials are shown in Figure 11 .

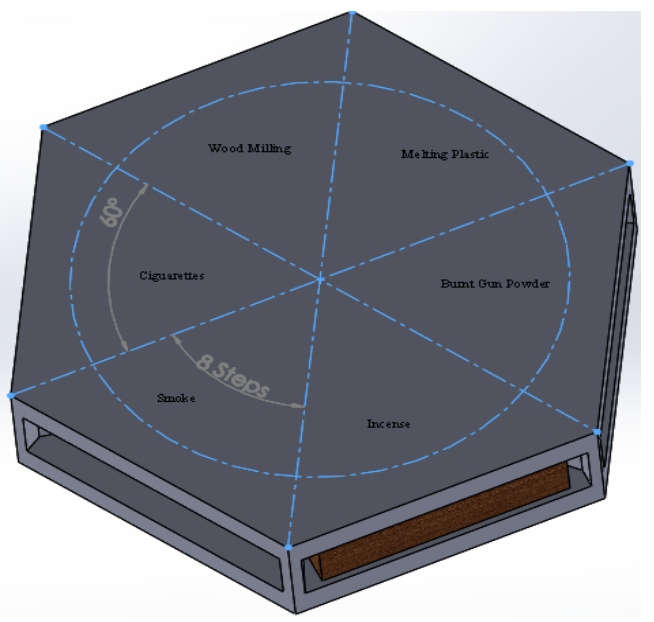

\section{Figure 11. Hexagon Faces and Number of Steps with Scent/odor Materials Order}

The stepper motor is a 48 steps per revolution giving a 7.5 degrees per step, it has a top speed of about 240 Rpm this gives:

Stepper speed $=250 \mathrm{Rpm}$

Revolution $=48$ Steps

$$
\text { Step time }=1 / \mathrm{Hz}=\frac{1}{\frac{240 \times 48}{60 \times 1000}}=5 \mathrm{~ms}
$$

Since the hexagon contains six faces, it requires the stepper to achieve 8 steps to turn the face of the stepper; the initial position of the laser is in the center of a hexagon face. In best case scenario the next scent material ejection is the current face, which requires zero rotation time. While if the next scent material ejection is a left or right neighboring face then the time required is $8 \times 5 \mathrm{~ms}$ time for one step which is approximately $40 \mathrm{~ms}$. Algorithm 1 stated that it finds the number of steps to rotate the current face to a required face in both directions, so the worst case scenario will take place only if the requested face is the opposite face and it takes $3 \times 8 \times 5 \mathrm{~ms}=120 \mathrm{~ms}$ to reach that face. To generalize these two findings we obtain the following equation:

$$
\begin{aligned}
& \text { Hexagon rotary average time }=\frac{\text { bestcase }+ \text { worstcase }+ \text { neighboring case }}{3} \\
& \qquad=\frac{(0)+(3 \times 8 \times 5)+(8 \times 5)}{3} \cong 53 \mathrm{~ms}
\end{aligned}
$$

In Table 2, all the times combinations are calculated and placed in the table, the shaded cells represent a counterclockwise decision to take place to reduce rotation time of the rotary hexagon.

Table 2. Showing Times in ms Required to Travel from Every Hexagon Face to Any Other Face in the System

\begin{tabular}{|l|c|c|c|c|l|l|}
\hline & $\begin{array}{c}\text { Wood } \\
\text { Milling }\end{array}$ & $\begin{array}{c}\text { Melting } \\
\text { plastic }\end{array}$ & $\begin{array}{c}\text { Gun } \\
\text { powder }\end{array}$ & Incense & Smoke & Cigarettes \\
\hline
\end{tabular}




\begin{tabular}{|c|c|c|c|c|c|c|}
\hline $\begin{array}{c}\text { Wood } \\
\text { Milling }\end{array}$ & 0 & 40 & 80 & 120 & 80 & 40 \\
\hline $\begin{array}{c}\text { Melting } \\
\text { plastic }\end{array}$ & 40 & 0 & 40 & 80 & 120 & 80 \\
\hline Gun powder & 80 & 40 & 0 & 40 & 80 & 120 \\
\hline Incense & 120 & 80 & 40 & 0 & 40 & 80 \\
\hline Smoke & 80 & 120 & 80 & 40 & 0 & 40 \\
\hline Cigarettes & 40 & 80 & 120 & 80 & 40 & 0 \\
\hline
\end{tabular}

Furthermore the rocking effect takes place each time a material is targeted by the laser. Leaving the two steps from each direction to avoid edges of hexagon leaving 6 steps to go back and forth for about one second to ensure better burning angle and focal point, if the laser is centering the face of the hexagon it means the degree is $90^{\circ}$ and each step is $7.5^{\circ}$ this means the laser will hit the solid scent material in the degrees $90^{\circ}, 97.5^{\circ}, 105^{\circ}, 112.5^{\circ}$ clockwise and $82.5^{\circ}, 75^{\circ}, 67.5^{\circ}$ counterclockwise with focal points variations between $\pm 4 \mathrm{~mm}$.

To include the human experiments into the experimental results, 20 participants were involved in this test to validate the smell delivery during game playing, the participant is placed $70 \mathrm{~cm}$ away the display's ejection point and assigning the right click to acknowledge the smell/odor is received. When the user is involved in the game and triggers a smell related event the software will start a timer and when he/she presses right click the timer stops indicating the time that the user detected a smell/odor. Table 3 shows the average times recorded for the 6 types of scents/odors materials. Table 3 also shows an assessment score from 1 to 10 given to the participants to tick the smell satisfaction level showing how much it is resembles a real world odor. In addition Figure 12 shows the actual olfactory display releasing a scent/odor material using the laser.

\section{Table 3. Human Participants Average Detection Times and Average Satisfaction Level for the Scent/odor}

\begin{tabular}{|c|c|c|}
\hline Smell/odor & $\begin{array}{c}\text { Human participants average } \\
\text { detection time }\end{array}$ & Smell satisfactory level \\
\hline Burnt gun powder & 3.60 & 7 \\
\hline Wood Milling & 3.73 & 8 \\
\hline Smoke & 3.84 & 8 \\
\hline Incense & 3.77 & 9 \\
\hline Cigarettes & 3.57 & 9 \\
\hline Melting plastic & 3.84 & 8 \\
\hline
\end{tabular}

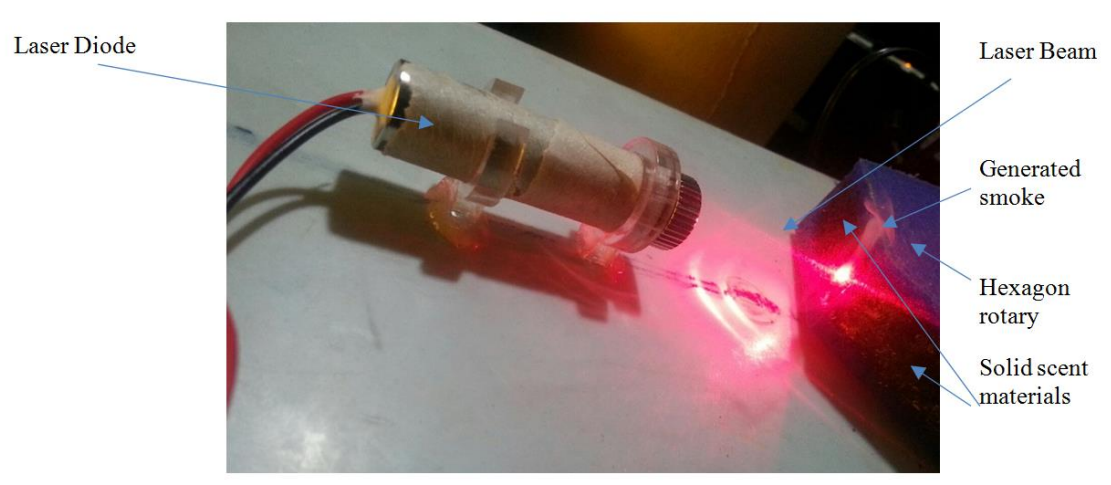

Figure 12. An Actual Image of the Laser Burning a Scent Material 


\section{Conclusions}

Building the proposed system and testing it resulted in fair amount of smell/odor presentation it was capable of delivering the exact smell type due to the usage of the original smell/odor materials, it is fair to state the cost is relatively low compared to producing a full operational system to satisfy the users and removing the fact that it needs additional cost for refilling the cartridges even for extensive usage. In terms of responsiveness against many scent related events it had shown a fair overall response time but still needs an additional improvements such as isolating recurrent events (burnt gun powder) into a separated cylindrical cartridge placed above the hexagon with separated laser diode to avoid interfering with other events and reducing the complexity of the software which increase response time. A control over the amount of smell is also achieved and shown a distinction in the provision of several levels of intensities that relates to the events inside the game. Finally user satisfaction is met according to the obtained results from users' feedback while using the actual device.

\section{Acknowledgment}

This work was partially supported by the National Natural Science Foundation of China (Grant No. 61173107), the National High Technology Research and Development Program of China (Grant No. 2012AA01A301-01), the Special Project on the Integration of Industry, Education and Research of Guangdong Province, China (Grant No. 2011A091000027) and the Project on the Integration of Industry, Education and Research of Huizhou, Guangdong Province, China (Grant No. 2012C050012012).

\section{References}

[1] T. Nakamoto and H. P. D. Minh, "Improvement of Olfactory Display Using Solenoid Valves", Proc. IEEE Virtual Reality, Charlotte, NC, March (2007), pp. 179-186.

[2] T. Nakamoto, M. Kinoshita, K. Murakami and A. Yossiri, " Demonstration of Improved Olfactory Display using Rapidly-Switching Solenoid Valves", IEEE Virtual Reality Conference, Lafayette, LA, (2009) March, pp. 301-302.

[3] T. Nakamoto and K. Yoshikawa, "Movie with Scents Generated by Olfactory Display Using Solenoid Valves", IEEE Virtual Reality Conference, (2006) March, pp. 291-292.

[4] H. Matsukura, H. Yoshida, H. Ishida, A. Saitoh, and T. Nakamoto,"Odor Presentation with a Vivid Sense of Reality: Incorporating Fluid Dynamics Simulation into Olfactory Display", IEEE Virtual Reality Conference, Lafayette, LA, (2009) March, pp. 295-296.

[5] M. Hirose, "A Study of Olfactory Display", Proc. of the Virtual Reality Society of Japan 2nd Annual Conference, in Japanese, (1997), pp. 155-158.

[6] T. Tanikawa, S. Tanaka, S. Sakikawa and M. Hirose, "A Study of Olfactory Display", Proc. of the Virtual Reality Society of Japan 5th Annual Conference, in Japanese, (2000), pp. 193-196.

[7] A. Mochizuki, T. Amada, S. Sawa, T. Takeda, S. Motoyashiki, K. Kohyama, M. Imura and K. Chihara, "Fragra: A Visual-Olfactory VR Game", ACM SIGGRAPH Sketches \& Applications, (2004).

[8] M. Göbel, "Trends in VR Display \& Interaction Technology", IEEE Virtual Reality Alexandria, Virginia, USA, (2006) March, pp. 122-125.

[9] R. McCarthy, "Scent-Emitting Systems", U.S. Patent 4,603,030, (1984).

[10] Y. Ariyakul and T. Nakamoto, "Olfactory Display Using a Miniaturized Pump and a SAW Atomizer for Presenting Low-volatile Scents", IEEE Virtual Reality Conference, Singapore, March (2011), pp.193194.

[11] A. Kadowaki, J. Sato, Y. Bannai and K. Okada, "Presentation Technique of Scent to Avoid Olfactory Adaptation", IEEE 17th International Conference on Artificial Reality and Telexistence, Esbjerg, Jylland, (2007) November, pp. 97-104.

[12] S. M. Kim and M. Y. Sung, "A Haptic Gaming System for Tactile Textures and 3D Shapes Discrimination" International Journal of Multimedia and Ubiquitous Engineering vol. 9, no. 9, (2014), pp. 319-334.

[13] S. Nizar Baharom, W. H. Tan and M. Z. Idris, "Emotional Design for Games: A Framework for PlayerCentric Approach in the Game Design Process" International Journal of Multimedia and Ubiquitous Engineering, vol. 9, no. 10, (2014), pp. 387-398. 


\section{Authors}

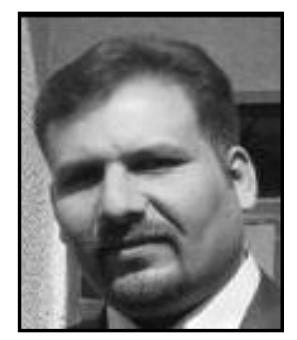

Saad Hameed, he was born in Baghdad - Iraq in 7 June 1979, he received his B.Sc. Degree in computer science at AL-Mansour University College 2001, and Masters Degree in computer Sciences, Iraqi committee for computer and informatics in 2004, he continued working in academic teaching in AL-Mansour University College for 11 years, through that time all of his research was self-funded and concentrated in automation and control, he has been promoted from assistant Lecturer to Lecturer in 2011, he is now a Ph.D. degree student at Hunan University in P.R. China.

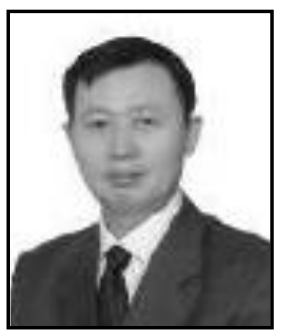

Zhiyong Li, he received the MSc degree in System Engineering from National University of Defense Technology, Changsha, China, in 1996 and PhD degree in Control Theory and Control Engineering from Hunan University, Changsha, China, in 2004. Since 2004, he joined the College of Information Science and Engineering of Hunan University. Now, he is a Full Professor with Hunan University, member of China Computer Federation (CCF). His research interests include visual object tracking, embedded computing system, dynamic multi-objective optimization evolutionary algorithm and tasks scheduling optimization in cloud computing. He has published more than 50 papers in international and domestic journals and conferences.

Prof. Li obtained several awards from academic organizations and conferences, such as the Champion of the Future Challenge: Intelligent Vehicles and Beyond, FC'09, which was hosted by the National Natural Science Fund Committee of China in 2009.

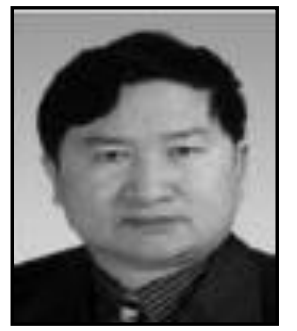

Renfa Li was born in Hunan - China in 1957, he works as Professor and PhD supervisor of Hunan University, Senior member of China Computer Federation (CCF), his main research interests include embedded system, artificial intelligence and optimization theory etc.

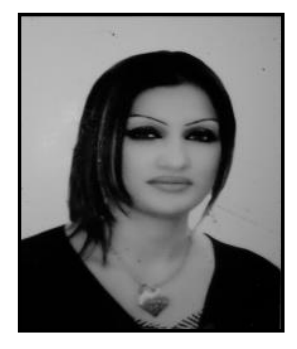

Jumana Waleed, she is a Ph.D. student in the School of information science and Engineering at Central South University, Changsha, China. Her research activity focuses on image processing, and information security working on digital watermarking. She received the B.S. degree in computers sciences from the Al-Yarmouk University College, Iraq, in 2004, and the M.S. degree in Computer Science/Data Security from the University of Technology, Baghdad, Iraq, in 2009. 